\title{
Preparation of microencapsulated $\alpha$-olefin drag reducing polymer used in oil pipeline transportation
}

\section{Li Bing', Xing Wenguo ${ }^{1}$, Dong Guilin ${ }^{1}$, Chen Xiangjun ${ }^{2}$, Zhou Ningning ${ }^{3}$, Qin Zhanbo ${ }^{4}$ and Zhang Changqiao ${ }^{1 *}$}

\author{
${ }^{1}$ School of Chemistry and Chemical Engineering, Shandong University, Shandong 250061, China \\ ${ }^{2}$ Linyi Institute of Product Quality Supervision and Inspection, Shandong 276004, China \\ ${ }^{3}$ Shandong Institute of Metrology, Shandong 250014, China \\ ${ }^{4}$ Shandong Quality Certification Centre, Shandong 250014, China
}

(C) China University of Petroleum (Beijing) and Springer-Verlag Berlin Heidelberg 2011

\begin{abstract}
Microcapsules containing oil drag-reducing polymer particles were prepared by meltingscattering and condensing of polyethylene wax, in-situ polymerization of urea and formaldehyde, and interfacial polymerization of styrene respectively. The related processes were studied by a molecular dynamics simulation method, and molecular design of microcapsule isolation agent was carried out on the basis of the simulation. The technologies for preparing microencapsulated oil drag-reducing polymer particles were compared and the circulation drag reducing efficiency of the microencapsulated polymer particles was evaluated based on the characterization results and their dissolution properties. Molecular design of a microcapsule isolation agent suggests that $\alpha$-olefin polymer particles can be stably dispersed in water by using long-chain alkyl sodium salt surfactant which can prevent the agglomeration of $\alpha$-olefin polymer particles. The results of simulation of the adsorption process shows that the amount of alkyl sodium salt surfactant can directly affect the stability of microencapsulated $\alpha$-olefin polymer particles. and there must be a minimum critical amount of it. After characterization of the morphology by Scanning Electron Microscopy (SEM) and comparison of the static pressure stability, especially the conditions of reaction and technological control of microcapsules with different shell materials, microencapsulation of $\alpha$-olefin polymer particles with poly-(urea-formaldehyde) as shell material was selected as the optimum scheme, because it can react under mild conditions and its technological process can be controlled in a large range. The relationship of drag reducing rate and dissolving time of microcapsules showed that the formation of microcapsules did not affect the maximum drag reducing rate, and the drag reducing rate of each sample can reach about $35 \%$ along with the dissolving time, i.e. microencapsulation did not affect the drag reducing property of $\alpha$-olefin polymer.
\end{abstract}

Key words: $\alpha$-olefin polymer, molecular design, coating material, microcapsule preparation, stability and solubility, drag reducing property

\section{Introduction}

As one of the five primary transportation modes (railway, road, water, airway, pipeline), pipeline transportation is a major transportation mode used in the petroleum industry, and drag reduction is one key factor in pipeline transportation because it affects the pipeline construction and operation costs and even pipeline safety (Cuenca et al, 2008; Li et al, 2000). Up to now, this technology is widely applied in oil transportation through adding drag reducing agent (DRA) in oil pipelines (Hu, 1997; Pang and Wei, 2010; Xu et al, 2010).

*Corresponding author. email: zhangchqiao@sdu.edu.cn Received June 10, 2010
Poly- $\alpha$-olefin, one of the macromolecular polymer drag reducing agents, plays a dominant role in drag reduction for oil pipeline transportation (Guan et al, 2001; Bhagat and Rochester, 1974; John et al, 1972; Burger et al, 1980; Ram and Kadim, 1970; Meier et al, 1974). This drag reducing polymer is commonly prepared by random copolymerization of more than 3 types of long-chain $\alpha$-olefins, and its molecular weight exceeds $6 \times 10^{6}$ (Wang and Dai, 1990). Due to the strong van der Waals forces among groups and entanglements among flexible chains, $\alpha$-olefin polymer is not crystalline at room temperature, but in a viscoelastic state (Song et al, 2000), which makes it can not be directly injected in oil pipelines. For the purpose of injection, $\alpha$-olefin polymer must be made into a suspension by a dispersion method. 
The dispersion method used at present is as follows: $\alpha$-olefin polymer in a glassy state at low temperature is crushed (Milligan and Smith, 2000; Labude et al, 2004), it is then dispersed into an organic dispersant, in which an isolation agent has been added in a low temperature environment to prepare a suspension with an $\alpha$-olefin polymer content of about $25 \mathrm{wt} \%$. However, this dispersion method can not effectively solve the problem about isolation of poly- $\alpha$-olefin particles, because the poly- $\alpha$-olefin particles in suspension often stick together during storage. This means the suspension can not be kept stably for a long time before being injected into the oil pipeline. In addition, the suspension with only 25 $\mathrm{wt} \% \alpha$-olefin polymer increases the cost of oil transportation. In recent years, microencapsulation enables the long term storage as solids of viscoelastic $\alpha$-olefin polymer powders. The microcapsules can be converted to a suspension on site just before use. As well as overcoming storage problems, this reduces the transportation cost (Burger et al, 1980; Macedo and Maneschy, 2000; Gyr and Tsinober, 1997). Avoiding agglomeration of viscoelastic $\alpha$-olefin polymer powders and stably storing it as isolated particles for a long time is the aim of the study.

In this paper, molecular dynamics simulation was used for studying related processes, and molecular design of the microcapsule isolation agent was carried out on the basis of the molecular dynamics simulation. Microencapsulation of oil drag reducing polymer particles were studied by melting-scattering and condensing polyethylene wax, in situ polymerization of urea and formaldehyde, and interfacial polymerization of styrene.In situ polymerization was determined as the optimum method for microcapsule preparation. As well, the circulation drag reducing efficiency of the microencapsulated polymer particles was evaluated.

\section{Molecular design of microcapsule isolation agent for $\alpha$-olefin drag reducing polymer}

For microencapsulation of $\alpha$-olefin drag reducing polymer, $\alpha$-olefin polymer must be first cooled to glassy state, then ground into particles and dispersed into water, in which an isolation agent was added, under vigorous stirring at low temperature. Because of the combined effects of the isolation agent and vigorous stirring, a stable suspension of $\alpha$-olefin drag reducing polymer particles was obtained. Then microencapsulation of $\alpha$-olefin drag reducing polymer particles was carried out in water. Generally, the isolation agent used in water is an anionic organic surfactant, which is called the $\alpha$-olefin drag reducing polymer microcapsule isolation agent.

The main role of the surfactant used as the isolation agent is to ensure that $\alpha$-olefin drag reducing polymer particles can disperse uniformly and stably in water. So the surfactant isolation agent should have the following properties: 1) it can effectively disperse $\alpha$-olefin drag reducing polymer particles uniformly in water; 2 ) it must be amphiphilic, i.e. it can be effectively absorbed on the surface of poly- $\alpha$-olefin particles to keep them stable in water system to prevent the particles agglomerating; 3 ) it should not react with poly- $\alpha$-olefin particles in the subsequent coating and microencapsulation.
Because of the variety of surfactants, molecular design of the isolation agent for $\alpha$-olefin drag reducing polymer is necessary in order to obtain a suitable surfactant. With the allatom molecular dynamics simulation method and COMPASS force field, discovery module of molecular simulation (MS) program was used to conduct energy minimization and molecular dynamics simulation calculation for the system. The energy minimization calculation was carried out first and then the molecular dynamics simulation calculation was performed. Based on the investigation of dynamic behaviors of several types of anionic and cationic surfactants by calculation and simulation, the behaviors of adsorption and agglomeration of long-chain alkyl sodium sulfate and sodium sulfonate in water were studied.

Fig. 1 shows the radial distribution of water molecules around two types of surfactant molecules. System B and system $\mathrm{C}$ are the distributions of water molecules around the hydrophilic parts (polar head) of long-chain alkyl sodium sulfate and sodium sulfonate, respectively. It can be seen from Fig. 1 that the water molecules distribution around hydrophilic parts of the two types of surfactant molecules is similar. The number of water molecules distributed around $\mathrm{O}_{1-3}$ is the largest. The difference between long-chain alkyl sodium sulfate and sodium sulfonate is that the number of water molecules surrounding $\mathrm{O}_{12}$ is the largest at radius $r=3.7$ $\AA$ for long-chain alkyl sodium sulfate molecules (system B), and there is a plateau at radius $r=3.8-5.0 \AA$ for the distribution of water molecules surrounding $\mathrm{O}_{12}$ for long-chain alkyl sodium sulfonate molecules. By integrating the curve, it is shown that the water amount around typical long-chain sodium sulfonate groups is larger than that around sodium sulfate groups, indicating that the sodium sulfonate groups have greater hydrophilic property than sodium sulfate groups. Fig. 2 shows the microdistribution of water molecules around polar heads. Fig. 2(a) shows the hydrogen bonding between polar parts of alkyl sodium sulfate and water molecules. Fig. 2(b) shows the distribution of the electron cloud of alkyl sodium sulfate molecules. Fig. 2(c) and (d) are the distribution of water molecules around polar heads of alkyl sodium sulfonate and alkyl sodium sulfate molecules, respectively. It can be seen that water molecules are distributed mainly

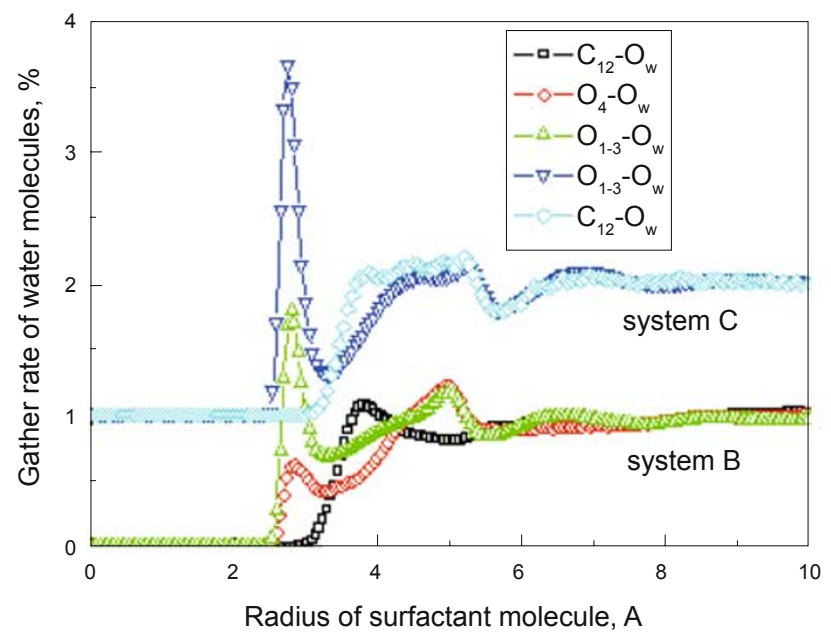

Fig. 1 The radial distribution of water molecules along two types of surfactant molecules 


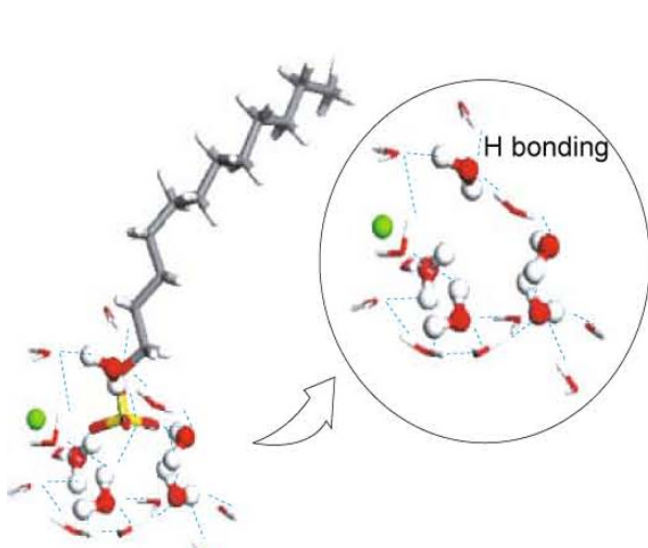

(a)

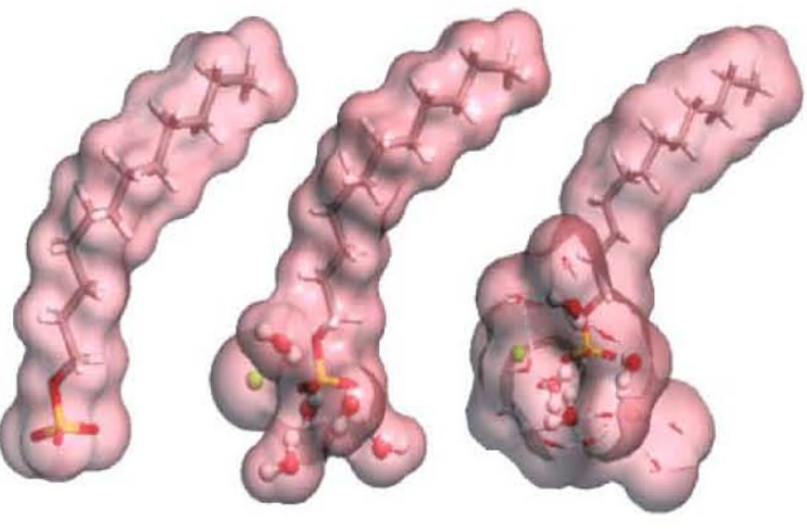

(b)

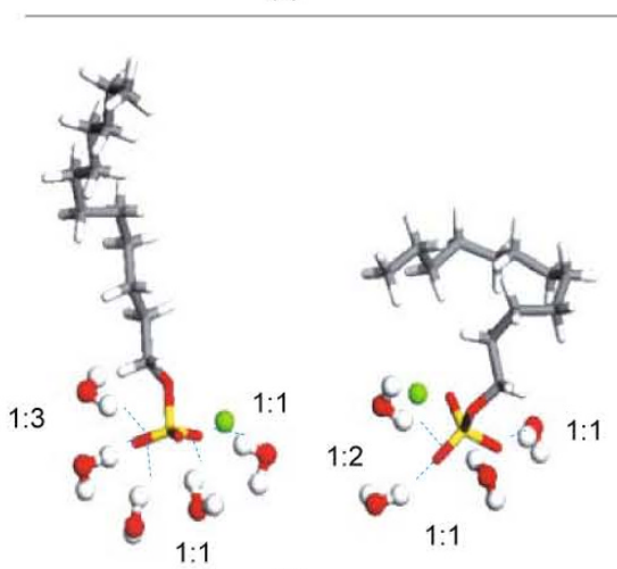

(c)

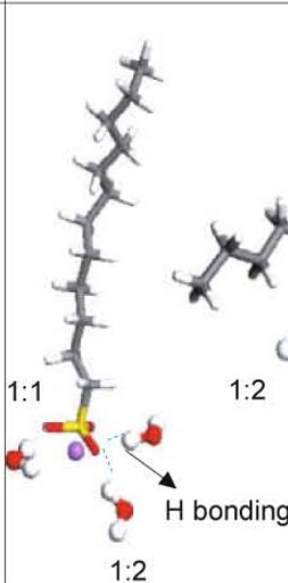

$1: 2$

Fig. 2 Microscopic distribution of water molecules near the polar head

around polar heads and there are almost no water molecules near the backbones of these two types of surfactants.

Charge distributions of water molecules around typical long-chain alkyl sodium sulfate and sodium sulfonate were calculated by two semi-empirical methods, PM3 and AM1, and are shown in Fig. 3 and Fig. 4 (y-axis is the charge strength, $\mathrm{x}$-axis is the distance between water molecule and sulfur atom). The calculation results show that when the distance between $\mathrm{S}$ atoms of long-chain alkyl sodium sulfate and water molecules is 2-2.5 $\AA$, the charge strength is the largest, then the charge strength decreases; while for long-chain alkyl sodium sulfonate, with increasing distance between $\mathrm{S}$ atoms and water molecules, the charge strength decreases gradually. But because these two surfactants both have long hydrophobic chains (longer than $10 \AA$ ) it can be predicted that their hydrophobic properties are almost the same.

For investigating the micro-interaction of $\alpha$-olefin polymer molecules and long-chain alkyl sodium sulfate, a 50 carbon-atom long-chain $\alpha$-olefin polymer and long-chain alkyl sodium sulfate as surfactant were used to calculate the interaction of their molecules. Fig. 5 and Fig. 6 show the simulated configuration of $\alpha$-olefin polymer molecules and long-chain alkyl sodium sulfate molecules after interaction, and the simulated dispersion of long-chain alkyl sodium sulfate molecules around $\alpha$-olefin polymer molecules,

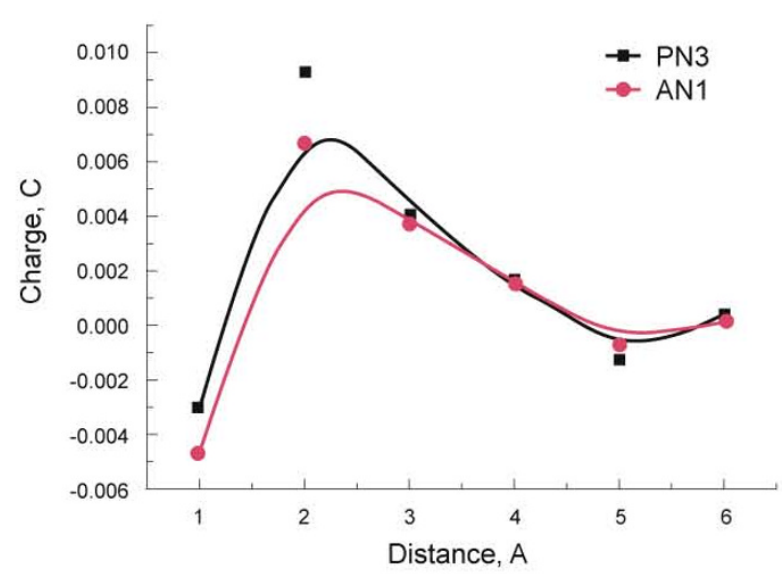

Fig. 3 The charge distribution of water molecules around long-chain alkyl sodium sulfate

respectively. The simulated calculation results show that there is a strong interaction between the hydrophobic part of longchain alkyl sodium sulfate and $\alpha$-olefin polymer molecules, and the hydrophilic group and polar part is exposed to water, and that the system will reach equilibrium with time. Fig. 7 and Fig. 8 show the molecular dynamic simulation of $\alpha$-olefin polymer in the adsorption process in the presence of long-chain alkyl sodium. It can be seen that the amount of surfactant has a direct effect on adsorption results. 


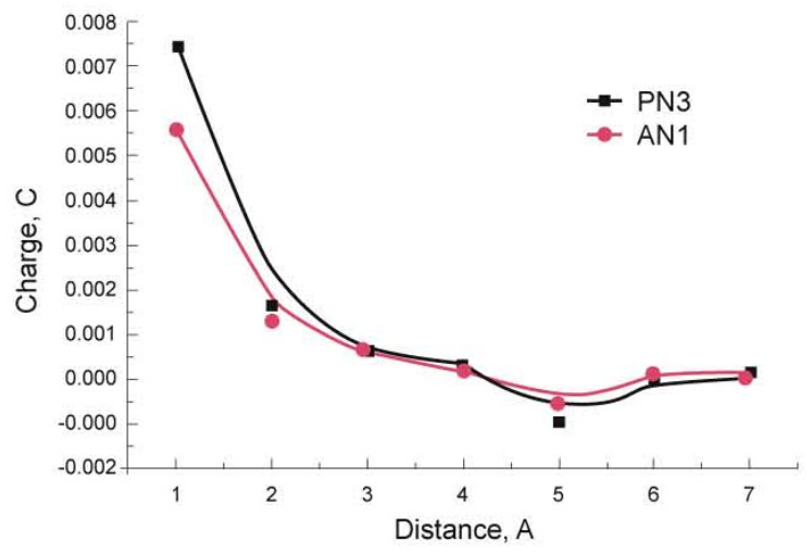

Fig. 4 The charge distribution of water molecules around long-chain alkyl sodium sulfonate

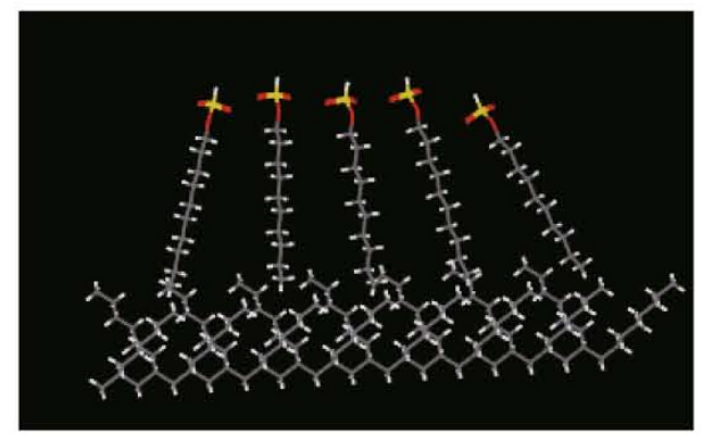

Fig. 5 The simulated configuration of $\alpha$-olefin polymer molecules and long-chain alkyl sodium sulfate after interaction Notes: The colorful part with white branched chain represents the long-chain alkyl sodium sulfate molecules, and the white part on the bottom represents $\alpha$-olefin polymer molecules

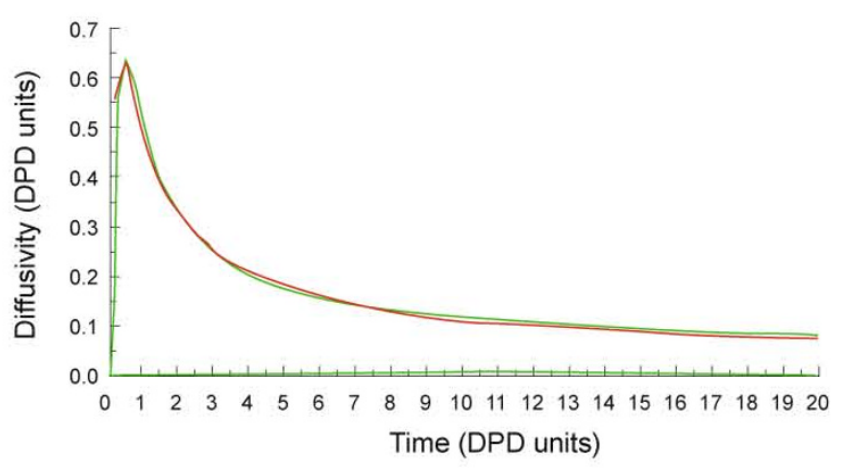

Fig. 6 The simulated dispersion of long-chain alkyl sodium sulfate around $\alpha$-olefin polymer molecules

(DPD units is dissipative particle dynamics units)

All the molecular dynamic simulation results mentioned above show that long-chain alkyl sodium. has excellent amphiphilic property, i.e. the surfactant can be absorbed effectively on the surface of $\alpha$-olefin polymer particles.

This property keeps $\alpha$-olefin polymer particles stable in suspension in water and further keeps them from agglomerating. Simultaneously, the simulation results of the $\alpha$-olefin polymer adsorption process indicate that an additional amount of surfactant has a direct effect on the

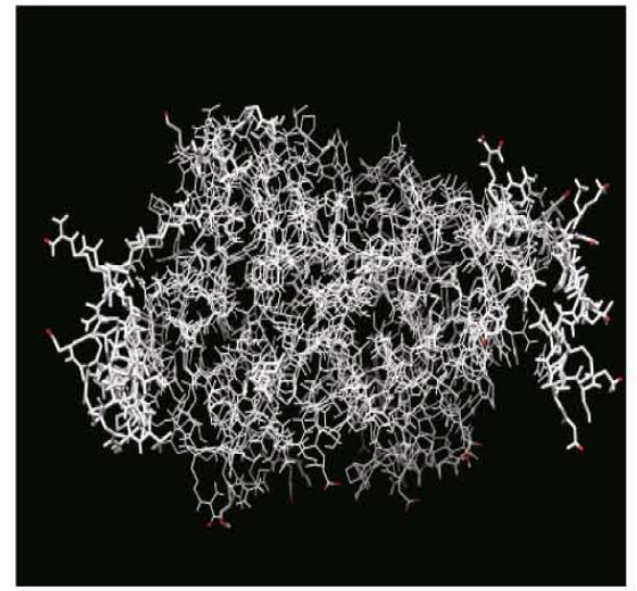

Fig. 7 Simulation of interaction of $\alpha$-olefin polymers and a small amount of surfactant

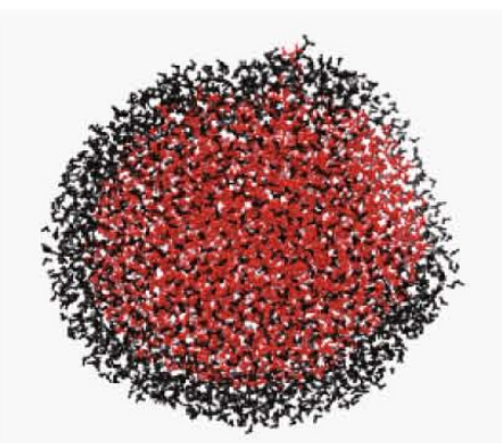

Fig. 8 Simulation of interaction of $\alpha$-olefin polymers and a large amount of surfactant

Notes: The red part represents the $\alpha$-olefin polymer molecules and the black part represents long-chain alkyl sodium sulfate

stability of $\alpha$-olefin polymer in water, and that there must be a minimal critical value for the amount of surfactant.

\section{Method for microencapsulation of $\alpha$-olefin drag reducing polymer}

From the microencapsulation principles, the methods for microencapsulating $\alpha$-olefin drag reducing polymer are classified into three types, namely chemical physico-chemical and mechanical (physical) methods (Xu, 2005). In this paper, three different oil drag reducing polymer microcapsules were prepared by interfacial polymerization (chemical method), in-situ polymerization (chemical method) and the meltingscattering and condensing method (physical method). The results of morphology characterization and storage stability of microcapsules showed that microcapsules prepared by in situ polymerization with urea and formaldehyde as shell materials were the best. In addition, evaluation of the circulation drag reducing efficiency of microcapsules prepared by these methods was performed.

\subsection{Experimental reagents}

Urea (AR, Tianjin Outer Ring Chemical Company Limited); formaldehyde solution (37 wt\%, Tianjin Duoli Fine Chemical Company Limited); dodecyl sodium sulfate (AR, Tianjin Development Zone Letai Chemical Company 
Limited); Sulfuric acid (98 wt\%, Shandong Huaxiang Industry Foreign Trade Development Company Limited); pH test paper (1-14); Filter screen (300 mesh); $\alpha$-olefin polymer powder (self-made); n-butanol (AR, Tianjin Outer Ring Chemical Company Limited); Ethylene glycol monomethyl ether (AR, Shandong Huaxiang Industry Foreign Trade Development Company Limited); Polyethylene wax (molecular weight: $\mathrm{M}_{\mathrm{w}}=2000$ ); Persulfate potassium (AR, Beijing Chemical Reagent Company Limited); Styrene (AR, Tianjin Benchmark Chemical reagent Co., Ltd.). All the reagents were used directly.

\subsection{Preparation of microcapsules}

\subsubsection{Preparation of oil drag reducing polymer} microcapsules by melting-scattering and condensing method

About $52 \mathrm{~g}$ n-butanol and $33 \mathrm{~g}$ ethylene glycol monomethyl ether were mixed in a flask and used as a dispersant (boiling point about $123{ }^{\circ} \mathrm{C}$ ). Then $2.25 \mathrm{~g}$ polyethylene wax (equivalent to $15 \mathrm{wt} \%$ of drag reducing polymer) was added to the dispersant and mildly heated under stirring (300 rpm). Polyethylene wax melted when the temperature was close to $123^{\circ} \mathrm{C}$. The vapor was refluxed back into the flask with a condenser. The suspension of drag reducing polymer particles stabilized by dodecyl sodium sulfate elaborated on above chapter in water was filtered. On the melting state of polyethylene wax, about $15 \mathrm{~g}$ of drag reducing polymer particles were added into the mixed dispersant, and then the stirring speed was decreased to be $100 \mathrm{rpm}$ and the temperature was decreased to $20^{\circ} \mathrm{C}$ in $10 \mathrm{~min}$ with a water bath. Finally the microcapsules were filtered and dried for $24 \mathrm{~h}$ at $60{ }^{\circ} \mathrm{C}$ in an oven, then crushed to obtain oil drag reducing polymer microcapsules coated with polyethylene wax.

3.2.2 Preparation of oil drag reducing polymer microcapsules by in-situ polymerization of ureaformaldehyde

To a flask about $170 \mathrm{~g}$ water and $0.2 \mathrm{~g}$ dodecyl sodium sulfate were added under stirring at $500 \mathrm{rpm}$. Then $5 \mathrm{~g}$ urea and $11 \mathrm{~g}$ formaldehyde solution were added directly and stirred for $5 \mathrm{~min}$, and then $60 \mathrm{~g}$ of oil drag reducing polymer powder was added. The mixture reacted for $10 \mathrm{~min}$ under stirring, then sulfuric acid was added until the $\mathrm{pH}$ was below 1 and stirring was continued for $30 \mathrm{~min}$. The polymer particles were filtered, and washed with deionized water until $\mathrm{pH}$ of the wash water was 7 . The drag reducing polymer particles were dried for $24 \mathrm{~h}$ at $60{ }^{\circ} \mathrm{C}$ then crushed to obtain oil drag reducing polymer microcapsules.

3.2.3 Preparation of oil drag reducing polymer microcapsule by styrene polymerization method

About $85 \mathrm{~g}$ water and $0.1 \mathrm{~g}$ dodecyl sodium sulfate were added in a flask under stirring at $500 \mathrm{rpm}$. Then about $0.1 \mathrm{~g}$ potassium persulfate was added to the flask. After stirring for $10 \mathrm{~min}, 4.5 \mathrm{~g}$ styrene, and $30 \mathrm{~g}$ drag reducing polymer particles was added and stirring continued for $10 \mathrm{~min}$. Then the stirring speed was decreased to 100 $\mathrm{rpm}$, and the temperature was raised to $80{ }^{\circ} \mathrm{C}$. Under this condition, reaction took place for $60 \mathrm{~min}$. The temperature was decreased to room temperature, and the drag reducing polymer particles were filtered, dried for $24 \mathrm{~h}$ at $60{ }^{\circ} \mathrm{C}$, and crushed to obtain oil drag reducing polymer microcapsules coated with polystyrene.

\section{Characterization and results analysis}

\subsection{SEM morphology}

The morphologies of oil drag reducing polymer microcapsules prepared by different methods and oil drag reducing polymer particles uncoated were characterized with a scanning electron microscope (SEM) (JEOL JSM-7600F, Japan) and example images are shown in Fig. 9.

It is found that the morphologies of drag reducing polymer particles showed significant change before and after coating. The surface of uncoated particles was random, while the surface of the coated with poly ethylene wax showed a smooth bulk composite with a layer-tablet structure on the surface. The particles coated with poly (urea-formaldehyde), appeared as stacked small balls. The particles coated with poly styrene, were similar to poly-(urea-formaldehyde) coated particles, but the globular units were larger, rough and nonuniform and exhibited adhesion between them.

\subsection{Measurement of static pressure stability of microcapsules}

$\alpha$-olefin drag reducing polymer microcapsules are usually stored in a vessel. The long-term static pressure, resulting from the stacking of microcapsules, directly affects the stability of the microcapsules. So the compressive resistance of microcapsules is an important factor for evaluation of the stability of microcapsules.

The experiment for the static pressure stability of microcapsule was carried out as follows: Different weights were added on top of the stacked drag reducing polymer microcapsules, and the microcapsules were sampled every 2 months. Then the relationship of dissolving time and viscosity of oil dissolving microcapsules samples was investigated, because it was related to the static pressure stability of microcapsules.

Three types of microcapsules were separately packed in a $10 \mathrm{~cm}$ square vessel with the height of the packed microcapsules being $1 \mathrm{~cm}$. A $10 \mathrm{~cm}$ square plate was placed on top of the packed microcapsules. Different weights (including the plate weight), which are equivalent to the weights of $12 \mathrm{~cm}, 24 \mathrm{~cm}, 36 \mathrm{~cm}, 48 \mathrm{~cm}$ and $60 \mathrm{~cm}$ thickness of drag reducing polymer microcapsules, were placed on the plate and the temperature was kept at $20{ }^{\circ} \mathrm{C}$. Table 1 shows the relationship between the accumulation degree of microcapsules and the equivalent packed microcapsules heights, corresponding to the different weights.

It can be seen from Table 1 that the drag reducing polymer particles coated with poly-styrene have absolutely solidified in the first sampling ( 2 months). In addition, it was very difficult for the massive polymer mass to be crushed into powders. This phenomenon indicates that the compressive resistance of poly-styrene coated microcapsules is too poor for commercial application. 

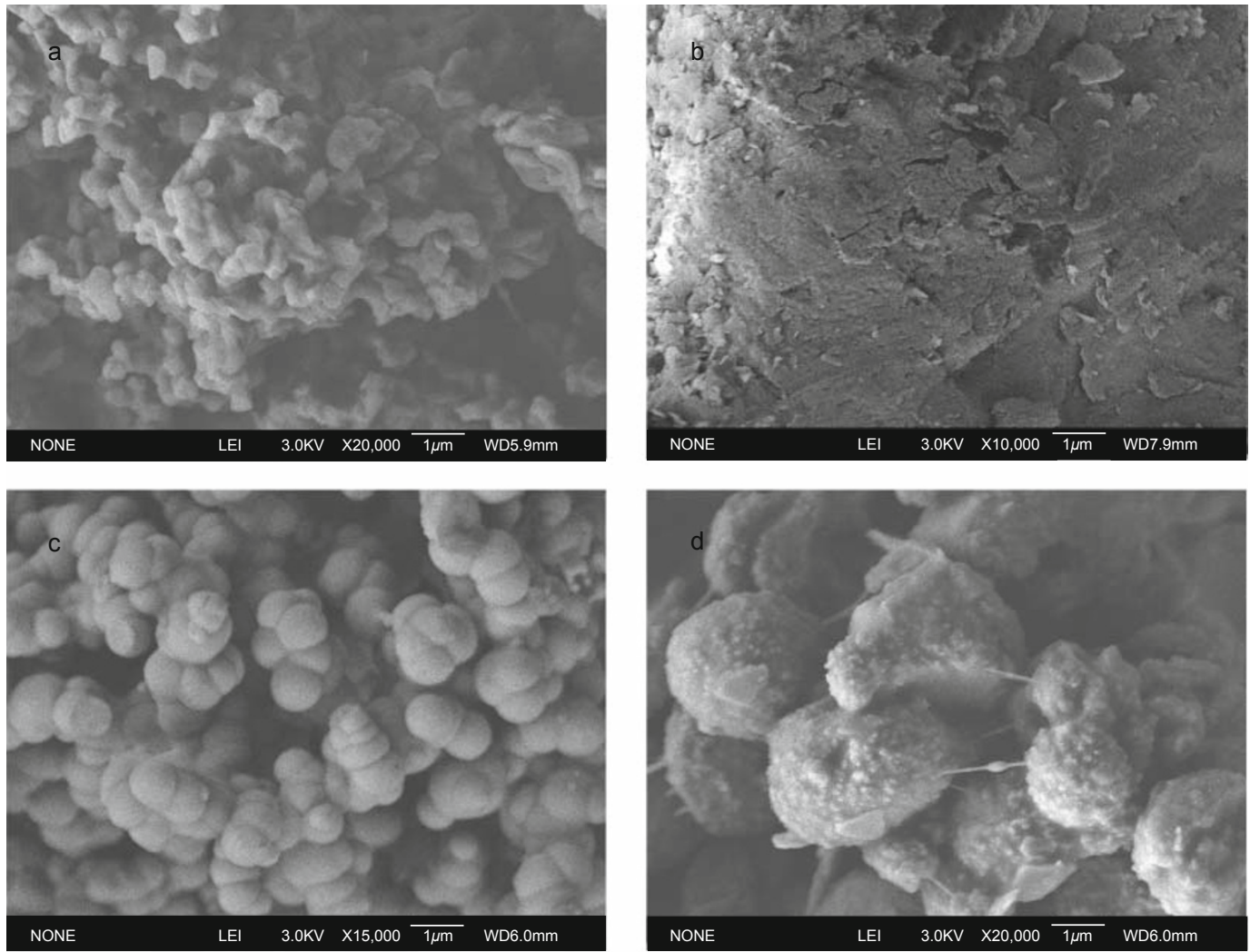

Fig. 9 SEM photograph of surface of oil drag reducing polymer particles and microcapsules

(a) uncoated drag reducing polymer particles, (b) polyethylene wax microcapsules, (c) poly-(urea-formaldehyde) microcapsules and (d) polystyrene microcapsules

Table 1 The relationship of accumulation degree of microcapsules with weights (cm) and time (month)

\begin{tabular}{|c|c|c|c|c|c|c|c|c|c|c|c|c|c|c|c|c|c|c|}
\hline \multirow{3}{*}{$\begin{array}{c}\text { Weights in terms of height } \\
\text { of microcapsules } \\
\mathrm{cm}\end{array}$} & \multicolumn{18}{|c|}{ Oil drag reducing agent microcapsules } \\
\hline & \multicolumn{6}{|c|}{ Coated with poly-ethylene wax } & \multicolumn{6}{|c|}{$\begin{array}{c}\text { Coated with } \\
\text { Poly-(urea-formaldehyde) }\end{array}$} & \multicolumn{6}{|c|}{ Coated with poly-styrene } \\
\hline & $2 \mathrm{~m}$ & $4 \mathrm{~m}$ & $6 \mathrm{~m}$ & $8 \mathrm{~m}$ & $10 \mathrm{~m}$ & $12 \mathrm{~m}$ & $2 \mathrm{~m}$ & $4 \mathrm{~m}$ & $6 \mathrm{~m}$ & $8 \mathrm{~m}$ & $10 \mathrm{~m}$ & $12 \mathrm{~m}$ & $2 \mathrm{~m}$ & $4 \mathrm{~m}$ & $6 \mathrm{~m}$ & $8 \mathrm{~m}$ & $10 \mathrm{~m}$ & $12 \mathrm{~m}$ \\
\hline 12 & $\sqrt{ }$ & $\sqrt{ }$ & $\sqrt{ }$ & $\sqrt{ }$ & $\sqrt{ }$ & $\sqrt{ }$ & $\sqrt{ }$ & $\sqrt{ }$ & $\sqrt{ }$ & $\sqrt{ }$ & $\sqrt{ }$ & $\sqrt{ }$ & $\times$ & $x$ & $x$ & $\times$ & $x$ & $x$ \\
\hline 24 & $\sqrt{ }$ & $\sqrt{ }$ & $\sqrt{ }$ & $\sqrt{ }$ & $\sqrt{ }$ & $\sqrt{ }$ & $\sqrt{ }$ & $\sqrt{ }$ & $\sqrt{ }$ & $\sqrt{ }$ & $\sqrt{ }$ & $\sqrt{ }$ & $\times$ & $x$ & $x$ & $\times$ & $\times$ & $x$ \\
\hline 36 & $\sqrt{ }$ & $\sqrt{ }$ & $\sqrt{ }$ & $\sqrt{ }$ & $\sqrt{ }$ & $\sqrt{ }$ & $\sqrt{ }$ & $\sqrt{ }$ & $\sqrt{ }$ & $\sqrt{ }$ & $\sqrt{ }$ & $\sqrt{ }$ & $\times$ & $x$ & $x$ & $\times$ & $\times$ & $x$ \\
\hline 48 & $\sqrt{ }$ & $\sqrt{ }$ & $\sqrt{ }$ & $\sqrt{ }$ & $\sqrt{ }$ & $\sqrt{ }$ & $\sqrt{ }$ & $\sqrt{ }$ & $\sqrt{ }$ & $\sqrt{ }$ & ○ & ० & $\times$ & $x$ & $x$ & $x$ & $x$ & $x$ \\
\hline 60 & $\sqrt{ }$ & $\sqrt{ }$ & $\sqrt{ }$ & $\sqrt{ }$ & $\sqrt{ }$ & $\sqrt{ }$ & $\sqrt{ }$ & $\sqrt{ }$ & $\sqrt{ }$ & 0 & $\circ$ & $\circ$ & $x$ & $x$ & $x$ & $x$ & $\times$ & $x$ \\
\hline
\end{tabular}

Notes: $\sqrt{ }$ represents that there is no cementation between microcapsules; $\circ$ represents that there is slight cementation between microcapsules, but they can be scattered in dispersant (alcohol mixture); $\times$ represents that there is serious cementation between microcapsules.

"m" represents month

Microcapsules coated with poly-ethylene wax had been in excellent particle state for 12 months, and no agglomeration occurred between particles. For the microcapsules coated with poly-(urea-formaldehyde), the fifth (10 months) sampling occurred slight agglomeration when it was pressed with $48 \mathrm{~cm}$ height of microcapsules, and the fourth (8 months) sampling occurred slight agglomeration when it was pressed with $60 \mathrm{~cm}$ height of microcapsules, but they can be crushed into powders. The SEM photographs of microcapsules coated with poly-(urea-formaldehyde) and pressed by corresponding weight of $60 \mathrm{~cm}$ thickness of them are shown in Fig. 10, which shows that there is no obvious difference between these morphologies, only regularity of small balls gradually declined.

\subsection{Solubility of microcapsules in oil}

The $\alpha$-olefin drag reducing agent must be dissolved in oil quickly when it is injected into oil pipelines. When the microencapsulation method is used, due to the shell protection to the core materials, the solubility of shell material in oil is an important factor for judgment of drag reduction. For the poly- $\alpha$-olefin drag reducing system, the relationship between 

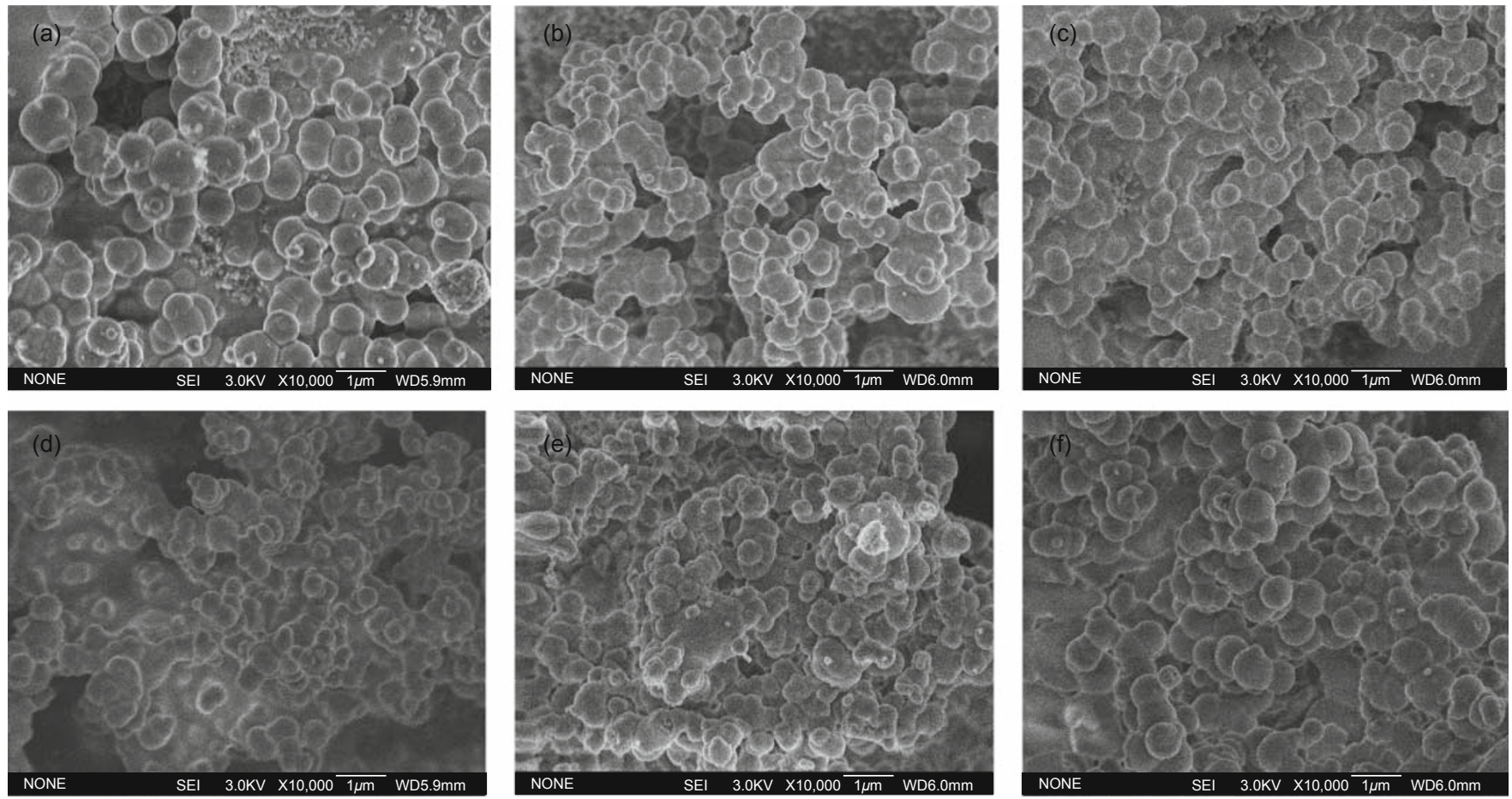

Fig. 10 The morphologies of microcapsules with poly-(urea-formaldehyde) resin coating (a) 2 months, (b) 4 months, (c) 6 months, (d) 8 months, (e) 10 months, (f) 12 months

the dissolving time of the $\alpha$-olefin drag reducing agent and its viscosity can indirectly represent the solubility of the microcapsules in oil.

The oil solubility of microcapsules coated with polyethylene wax and poly-(urea-formaldehyde) which has better static pressure stability was investigated. About $0.5 \mathrm{~g}$ uncoated drag reducing polymer particles or microcapsule samples were added to $500 \mathrm{ml}$ diesel and stirred at $500 \mathrm{rpm}$ until they dissolved absolutely, and the viscosities of the oil were measured every 10 min. Fig. 11 and Fig. 12 show the relationship of dissolving time and viscosity.

It can be seen from Fig. 11 and Fig. 12 that the increase in the viscosity of oil dissolving drag reducing polymer particles coated with poly ethylene wax was delayed compared with that dissolving uncoated particles, but their peak values were equivalent. After peak, the viscosity decreased slightly. The drag reducing polymer microcapsules coated with poly-(ureaformaldehyde) dissolved more rapidly than those coated with poly-ethylene wax, and the peak value appeared earlier. For the same microcapsule, the microcapsule dissolved more rapidly under large pressure (high weight) than under smaller pressure, and the peak appeared earlier.

\subsection{Evaluation of efficiency of oil drag reducing microcapsules}

The more rapid the microcapsules dissolve, the more effective the oil drag reducing polymer will be. The solubility of these two microcapsules in oil is slightly different. But in microencapsulation of drag reducing polymer particles with poly-ethylene wax, there was a melting and condensing process, and this microencapsulation process was carried out at the boiling point of mixed dispersant, which evaporates vigorously; however, preparation of microcapsules with poly- (urea-formaldehyde) as shell material was carried out in a mild reaction environment and the control conditions were flexible. From the practical application point of view, the microencapsulation method with poly-(urea-formaldehyde) as shell material seems the optimum scheme.

The drag reducing efficiency of the microcapsules with poly-(urea-formaldehyde) as shell material, after 6 months of static pressure stability experiment, was evaluated. Fig. 13 is the simulation circulation system for evaluation of drag reducing rate $(100 \mathrm{~L}$ diesel is used as the medium in this system). With this system, the increment in oil transportation rate $(F I \%)$ was calculated by measuring the volume flow of

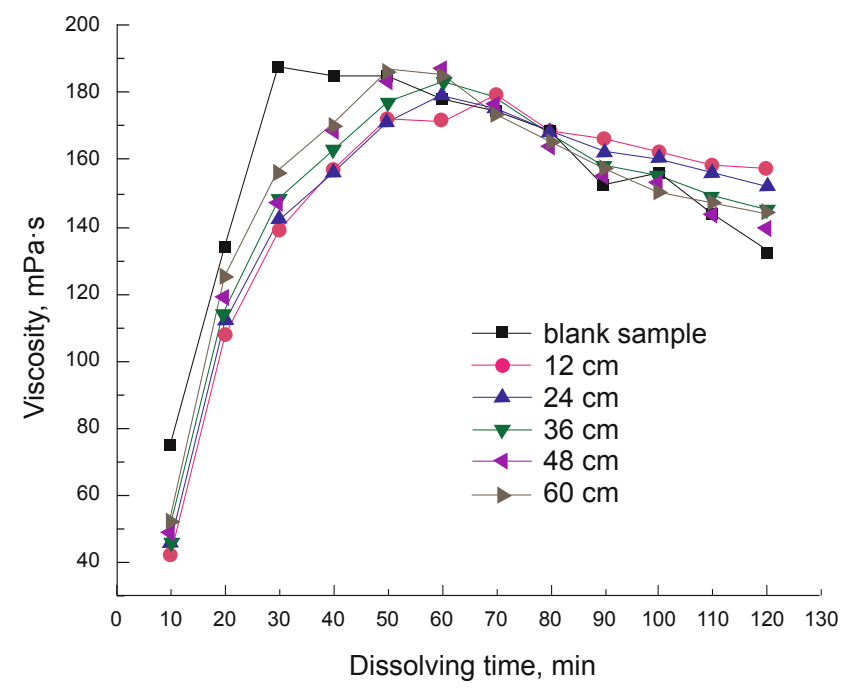

Fig. 11 Relationship of dissolving time and viscosity of microcapsules coated with poly ethylene wax

Notes: $12 \mathrm{~cm}, 24 \mathrm{~cm}, 36 \mathrm{~cm}, 48 \mathrm{~cm}, 60 \mathrm{~cm}$ represent weights in terms of height of microcapsules. The blank sample is uncoated polymer particles 


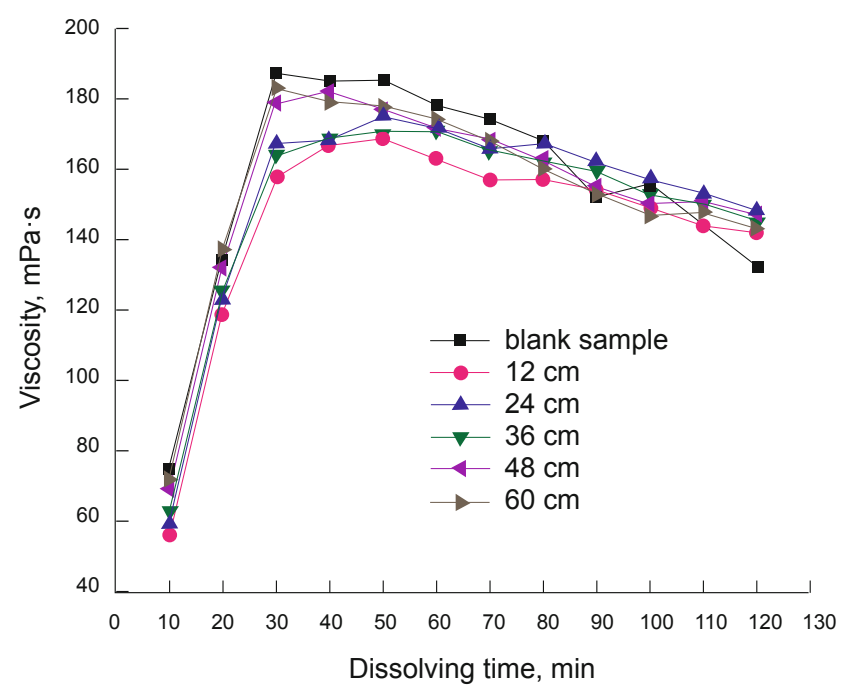

Fig. 12 Relationship of dissolving time and viscosity of microcapsules coated with poly-(urea-formaldehyde)

Note: $12 \mathrm{~cm}, 24 \mathrm{~cm}, 36 \mathrm{~cm}, 48 \mathrm{~cm}, 60 \mathrm{~cm}$ represent weights in terms of height of microcapsules. The blank sample is uncoated polymer particles

diesel before and after adding the drag reducing agent, then the drag reducing rate $(D R \%)$ was calculated by the following equation (An et al, 2008).

$$
F I \%=\left[\left(\frac{1}{1-\frac{D R \%}{100}}\right)^{0.55}-1\right] \times 100
$$

The relationship of dissolving time and drag reducing rate of microcapsules under different weights and of uncoated sample is shown in Fig. 14. It can be seen from Fig. 14 that the $\alpha$-olefin drag reducing polymer microcapsules under different weights had a similar relationship of drag reducing rate and dissolving time as the uncoated sample, i.e. the drag reducing rate increased to a maximum value and then decreased gradually. With the dissolving time, the drag reducing rates of microcapsule samples under different weights could reach about $35 \%$. So the microcapsule with shell material has little effect on drag reducing rate. Although the peak value of drag reducing rate of microcapsules

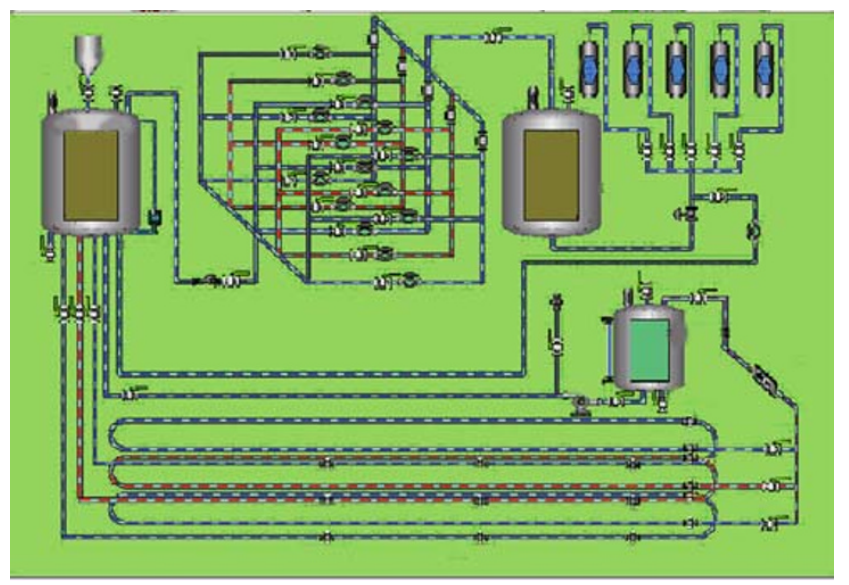

Fig. 13 Simulation circulation system for evaluation of oil drag reducing rate appeared later than that of uncoated sample, this delay could be shortened in practical oil transportation process due to the effect of high-Reynolds-number turbulence, so this delay has little effect on engineering applications. It can also be seen from Fig. 14 that after the peak value of about 35\%, all the drag reducing rates of microcapsules under different weights and of uncoated sample decreased slowly. This was resulted from the degradation of the drag reducing polymer, and was not related to microencapsulation.

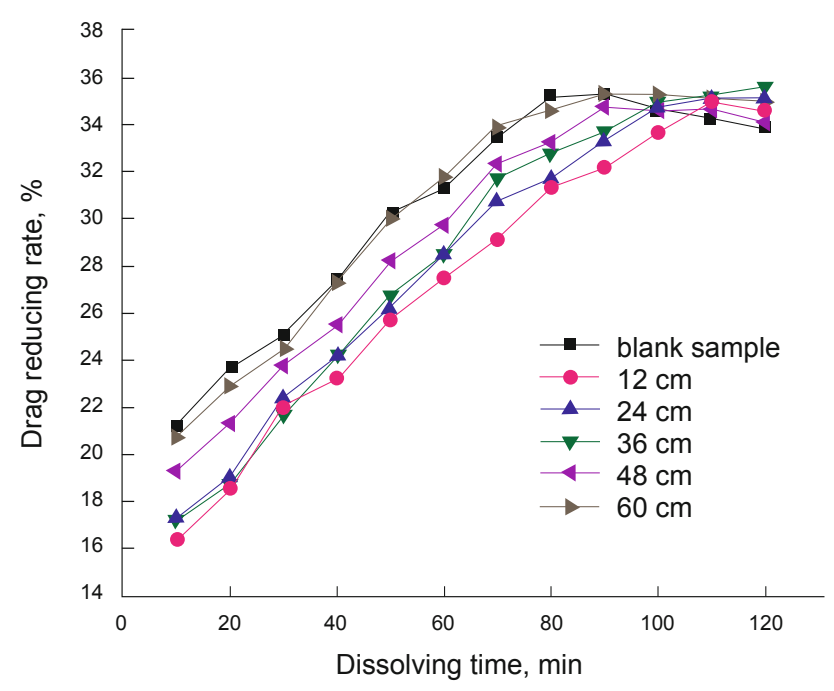

Fig. 14 Relationship of dissolving time and drag reducing rate of microcapsules under different weights and of uncoated sample (blank sample)

\section{Conclusions}

(1) The molecular design of the $\alpha$-olefin drag reducing polymer microcapsule isolation agent shows that, long chain alkyl sodium salts have excellent amphiphilic property that keeps $\alpha$-olefin polymer particles stable in water suspension. The simulation of adsorption process of $\alpha$-olefin polymer molecules shows there is a minimum critical amount of the long chain alkyl sodium salt surfactants and that the amount of surfactant directly affects the stability of $\alpha$-olefin polymer in water.

(2) Microcapsules containing oil drag reducing polymer were prepared by melting-scattering and condensing of polyethylene wax, in situ polymerization of urea and formaldehyde and interfacial polymerization of styrene. The SEM morphology characterization and static pressure stability of microcapsules were compared, and reaction conditions and methods were contrasted. Based on the above analysis, microencapsulation with poly-(urea-formaldehyde) as the shell material is recommended as the optimum scheme, because it can be carried out in mild condition and the process can be controlled over a wide range.

(3) The relationship of drag reducing rate and dissolving time of microcapsules pressed by different weights shows that, when effective concentrations of $\alpha$-olefin drag reducing polymer in different samples are the same, the change trends are consistent in drag reducing rate and dissolving time for microcapsules and uncoated sample, i.e. drag reducing rate increased to maximum point and then decreased gradually. 
Drag reducing rate of different samples can reach about 35\%. So the formation of microcapsules has little effect on drag reducing rate of $\alpha$-olefin drag reducing polymer.

\section{References}

An Q Q, Hu S J, Wang Y P, et al. Preparation of Mesoporous Silica SBA-15/hyperbranched polyurethane hybrids and its structure and properties. Journal of Materials Engineering. 2008. (10): 374-377 (in Chinese)

Bhagat G C and Rochester N Y. Rotary brush development. U.S. Patent. 1974. 3357402

Burger E D, Chorn L G and Perkins T K. Studies of drag reduction conducted over a broad range of pipeline conditions when flowing Prudhoe bay crude oil. Journal of Rheology. 1980. 24(5): 603-626

Burger E D, Munk W R and Wahl H A. Flow increase in the trans alaska pipeline flow using a polymeric drag reducing additive. SPE. 1980. 9419(9): 35-42

Cuenca F G, Marin M G and Diaz M B F. Energy-savings modeling of oil pipelines that use drag-reducing additives. Energy Fuels. 2008. 22(5): 3293-3298

Guan Z Y, Li G P, Zhao L Y, et al. A worldwide development in DRA study. Oil \& Gas Storage and Transportation. 2001. 20(6): 1-4 (in Chinese)

Gyr A and Tsinober A. On the rheological nature of drag reduction phenomena. J. Non-Newtonian Fluid Mech. 1997. 73(2): 153-162

Hu T N. Applied experiments on drag reducer in home oil transportation pipelines. Oil \& Gas Storage and Transportation. 1997. 16(6): 11-14 (in Chinese)

John D C, Rolla M and Gifford G M. Method of friction loss reduction in oleaginous fluids flowing through conduits. U.S. Patent. 1972.
3692676

Labude K M, Smith K W and Johnston R L. Drag reducing polymer suspensions. U. S. Patent. 2004. 6565053

Li G P, Yang R and Wang $\mathrm{K} \mathrm{H}$. The new research and production development of drag reducing agent in China and abroad. Oil \& Gas Storage and Transportation. 2000. 19(1): 3-7 (in Chinese)

Macedo E N and Maneschy C E. Analysis of the mass transfer entry region for drag-reducing viscoelastic fluids in turbulent pipe flow. Int. Comm. Heat Mass Transfer. 2000. 27(1): 59-68

Meier D J, Cerrito E C and Kruka V R. Method and composition for reducing the frictional drag of flowing fluids. U.S. Patent. 1974. 3801508

Milligan S M and Smith K W. Drag-reducing polymers and dragreducing polymer suspensions and solutions. U.S. Patent. 2000. 6576732

Pang M J and Wei J J. Progress in the study on turbulent flows of drag reducing surfactant solutions. Advances in Mechanics. 2010. 42(2): 129-146 (in Chinese)

Ram A and Kadim A. Shear degradation of polymer solutions. Journal of Applied Polymer Science. 1970. 14(8): 2145-2156

Song Z Z, Zhang X J and Ge J J. Research Situation of Crude Drag Reducing Agent. Oil-Gasfield Surface Engineering. 2000. 19(6): 7-9 (in Chinese)

Wang Y L and Dai J L. Drag reducing polymers: structure/property relationship. Oilfield Chemistry. 1990. 7(1): 98-106 (in Chinese)

$\mathrm{Xu} \mathrm{C}, \mathrm{Li} \mathrm{Y} \mathrm{S}, \mathrm{Wu} \mathrm{H} \mathrm{H}$, et al. Experimental study on drag reduction of surfactant. Oil \& Gas Storage and Transportation. 2010. 29(2): 124127 (in Chinese)

Xu Z H. Preparation of microcapsules and its application. New Chemical Materials. 2005. 33(11): 78-81 (in Chinese)

(Edited by Zhu Xiuqin) 